\title{
Expression profiling analysis reveals molecular mechanism of Lnc00675 downregulation promoting cell apoptosis in acute myeloid leukemia U937 cells
}

\author{
Miao Miao, Mengqi Li, Zhuogang Liu, Wei Yang, Chen Wang, Rong Hu \\ Department of Hematology, Shengjing Hospital, China Medical University, Shenyang, China \\ Contributions: (I) Conception and design: M Miao, R Hu; (II) Administrative support: R Hu; (III) Provision of study materials or patients: Z Liu, W \\ Yang; (IV) Collection and assembly of data: M Li, C Wang; (V) Data analysis and interpretation: M Miao; (VI) Manuscript writing: All authors; (VII) \\ Final approval of manuscript: All authors. \\ Correspondence to: Dr. Rong Hu. Department of Hematology, Shengjing Hospital, China Medical University, 39 Huaxiang Road, Shenyang, Liaoning \\ 110021, China. Email: hur@sj-hospital.org.
}

Background: Acute myeloid leukemia (AML), an aggressive malignancy with poor prognosis, is the most common in adult leukemia. Long non-coding RNA (lncRNA) could affect the regulation of protein-coding genes, cell proliferation and apoptosis, tumor cell resistance to radio- and chemotherapy and pathological processes. Lnc00675 is a lncRNA also known as transmembrane protein 238 like (TMEM238L), which identified as a marker of tumor promoter and unfavorable prognosis in patients with pancreatic ductal adenocarcinoma, glioma and cervical cancer. However, the association between Lnc00675 and hematological tumors has not been previously reported.

Methods: Expression profile gene chip technology was used to screen for differentially expressed genes (DEGs) through comparing Lnc00675 overexpression and Lnc00675 downregulation. Gene ontology (GO) analysis was performed to identify the biologic implications of the DEGs. Kyoto Encyclopedia of Genes and Genomes (KEGG) pathway analysis was performed to identify biologically important pathways associated with the DEGs. Cell Counting Kit-8 (CCK-8) assay and flow cytometric analysis were utilized to detect the cell proliferation rate and the cell apoptosis rate, respectively.

Results: Comparing Lnc00675 overexpression and Lnc00675 downregulation, a total of 866 and 1,115 DEGs were upregulated and downregulated, respectively. Bioinformatics analysis indicated that Lnc00675 might affect U937 cells proliferation and apoptosis through JAK-STAT signaling pathway and PI3K-Akt signaling pathway. The cell proliferation rate in si-Lnc00675 group was significantly lower than those of siNC group and Lnc00675 group $(\mathrm{P}<0.05)$. The cell apoptosis rate of si-Lnc00675 group $(22.93 \% \pm 2.24 \%)$ was significantly higher than those of si-NC group $(0.37 \% \pm 0.88 \%)$ and Lnc00675 group $(0.73 \% \pm 0.35 \%)$ $(\mathrm{P}<0.01)$.

Conclusions: Downregulation of lnc00675 expression inhibited proliferation and promoted apoptosis in human leukemia U937 cells.

Keywords: Lnc00675; U937 cells; acute myeloid leukemia (AML); JAK-STAT signaling pathway; PI3K-Akt signaling pathway; expression profile analysis

Submitted Mar 28, 2020. Accepted for publication Sep 26, 2020.

doi: $10.21037 /$ tcr-20-1714

View this article at: http://dx.doi.org/10.21037/tcr-20-1714 


\section{Introduction}

Acute myeloid leukemia (AML), an aggressive malignancy with poor prognosis, is the most common in adult leukemia. The therapeutic efficacy of patients with AML remains poor, with only $40 \%$ young patients ( $<60$ years old) or $10 \%$ patients ( $>60$ years old) achieving long-term survival (1). The pathological mechanism of AML is a series of events including changes in cell proliferation, differentiation, and apoptosis caused by pathogenic factors, such as somatic mutations, cytogenetic abnormalities, epigenetic changes $(2,3)$.

Long non-coding RNAs (lncRNAs) are e a class of RNAs longer than 200 nucleotides, which don't have the function of encoding proteins (4). LncRNA could affect proteincoding gene regulation, cell proliferation and apoptosis, tumor cell resistance to radio- and chemotherapy and pathological processes by participating in transcriptional regulation and post-transcriptional regulation (5-8). Accumulating evidence supports that misregulation of lncRNA-based epigenetic networks contribute to many types of cancer $(9,10)$. Lnc00675 is a lncRNA also known as transmembrane protein 238 like (TMEM238L), and is identified as a marker of tumor promoter and unfavorable prognosis in patients with pancreatic ductal adenocarcinoma (11), glioma (12) and cervical cancer (13). In spite of the aforementioned link between Lnc00675 and cancer, very few researches have been carried out to find the molecular mechanism of Lnc00675 in cancer metastasis. Li et al. reported the positively correlation between Lnc00675 expression and TRIP6 protein expression in glioma tissues and cell lines (12). Ma et al. reported that LINC00675 promoted cervical tumorigenesis by modulating the Wnt/ $\beta$-catenin pathway (13).

However, the association between Lnc00675 and hematological tumors has not been previously reported. In the current study, we analyzed the effect of Lnc00675 on proliferation and apoptosis in human leukemia U937 cells, and the other aim of the current study was to investigate molecular mechanism of Lnc00675 using expression profiling analysis. Our results probably identify Lnc00675 as a novel therapeutic target and provide a new perspective for molecular mechanisms of AML.

\section{Methods}

\section{Cell culture and transfection}

Human leukemia U937 cells (RRID: CVCL_0007) was cultured in 90\% RPMI-1640 (Hyclone, USA) + 10\% FBS (Gibco, USA) + penicillin (100 U/mL) and streptomycin $(100 \mathrm{~g} / \mathrm{mL})$. Cells were cultured under $5 \% \mathrm{CO}_{2}$ and $95 \%$ air in an incubator set at $37^{\circ} \mathrm{C}$. U937 cells in logarithmic growth phase were divided into three groups, such as Lnc00675 group, si-Lnc00675 group and si-NC group. U937 cells were seeded in $25 \mathrm{~cm}^{2}$ cell culture flasks.

\section{Cell transfection}

Transfections were performed using Lipofectamine ${ }^{\mathrm{TM}} 2000$ (Invitrogen, USA). U937 cells suspended in serum-free RPMI-1640 were inoculated in $25 \mathrm{~cm}^{2}$ cell culture flasks to undergo transfection with Lnc00675 overexpression vector (Lnc00675 group), Lnc00675 siRNA vector (si-Lnc00675 group), and Lnc00675 siRNA negative control vector (si-NC group), respectively. All nucleotide vectors were purchased from Shanghai Genechem Co., Ltd. (China).

\section{Microarray analysis}

U937 cells of Lnc00675 group and si-Lnc00675 were isolated, pelleted cells by centrifugation, respectively. Used $1 \mathrm{~mL}$ of TRIzol Reagent (Invitrogen, USA) to lyse $1 \times 10^{7}$ U937 cells by repetitive pipetting. Microarray experiments were conducted by Shanghai KangChen Biotech (China) with Agilent Human 4x44K Gene Expression Microarray chips with 444,000 probes, the Agilent One-Color Microarray-Based Gene Expression Analysis protocol was used, including total RNA Clean-up and RNA QC, purify the labeled/amplified RNA and labeled cRNA QC, hybridization, microarray Wash, Scanning, extract data using Agilent Feature Extraction software. Bioconductor DESeq2 version 1.12.3 (https://www.rdocumentation. org/packages/DESeq2) was used to identify differentially expressed genes (DEGs) using a fold change (FC) $>2$ for significant upregulation or significant downregulation and a false discovery rate $(\mathrm{FDR})<0.05$. A scatter plot was drawn according to the analysis of the DEGs. Gene ontology (GO, www.geneontology.org) analysis was performed to identify the biologic implications of the DEGs. Fisher's exact test was used to identify the significant GO terms with FDR-adjusted P values. Kyoto Encyclopedia of Genes and Genomes (KEGG) pathway analysis was performed to 


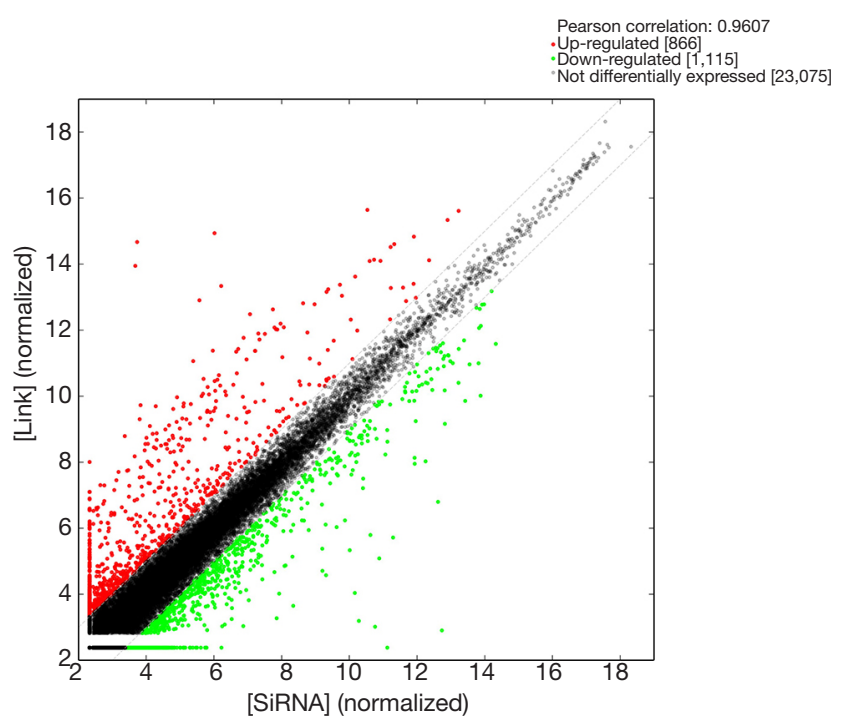

Figure 1 Scatter plot of upregulated and downregulated differentially expressed genes comparing between Lnc00675 group and si-Lnc00675.

identify biologically important pathways associated with the DEGs. Fisher's exact test was used to select the significant pathways based on $\mathrm{P}$ values $(\mathrm{P}<0.05)$ and FDR $(\mathrm{FDR}<0.27)$.

\section{Cell apoptosis detection by flow cytometry}

The cells of Lnc00675 group, si-Lnc00675 group and si-NC group were seeded in 6-well cell culture plate, respectively. After $48 \mathrm{~h}$ of transfection, the U937 cells were washed with PBS. Flow cytometry was used to detect the apoptosis rates of the three groups. The staining was performed by Annexin V-FITC/PI double staining method (GenStar, China). Binding buffer was used to resuspend cells, $5 \mu \mathrm{L}$ of Annexin V-FITC was added, then incubated at room temperature for 15 minutes in the dark. PI staining $(5 \mu \mathrm{L})$ was added for 5 minutes before detection. A FACS Calibur cell analyzer (BD Biosciences) was used to analyze cell apoptosis and apoptosis rate. The percentages of apoptotic cells including early apoptotic cells (Annexin $\mathrm{V}^{+} /$ $\mathrm{PI}^{-}$cells) and late stage apoptotic cells (Annexin $\mathrm{V}^{+} / \mathrm{PI}^{+}$ cells) were calculated.

\section{Cell Counting Kit-8 (CCK-8) assay}

The viability of U937 cells was detected using CCK-8 assay (Coffit, China). U937 cells $\left(1 \times 10^{5}\right.$ cells $\left./ \mathrm{mL}\right)$ in the logarithmic growth phase were prepared as cell suspensions using RPMI-1640 containing 10\% FBS. Cell suspension $(100 \mu \mathrm{L})$ was inoculated into a well of 96-well plates. 96well plate was incubated at $37{ }^{\circ} \mathrm{C}$ and $5 \% \mathrm{CO}_{2}$ for 24,48 or $72 \mathrm{~h}$ after transfection. CCK-8 solution $(10 \mu \mathrm{L})$ was added to each well and incubated for $2 \mathrm{~h}$ at $37^{\circ} \mathrm{C}$. The absorbance of each well was measured by microplate reader (Shanghai Flash Spectrum Biotechnology, China) at a wavelength of $450 \mathrm{~nm}$. The proliferation rate was calculated using the equation: proliferation rate $(\%)=\left(\mathrm{OD}_{\text {treatment }}-\mathrm{OD}_{\text {blank }}\right) /$ $\left(\mathrm{OD}_{\text {control }}-\mathrm{OD}_{\text {blank }}\right) \times 100 \%$.

\section{Statistical analysis}

GO and KEGG analyses were performed using the online database DAVID 6.8 (https://david.ncifcrf.gov/). The difference between 2 groups was determined by unpaired Student's $t$-test using GraphPad prism 8.0 software. The differences were considered statistically significant at $\mathrm{P}<0.05$. All experimental results are presented as the mean $\pm \mathrm{SD}$.

\section{Results}

\section{Differential gene expression}

By comparing Lnc00675 group with si-Lnc00675 group, the microarray analysis determined a total of 1,981 DEGs $(\mathrm{FC} \geq 2)$ (Figure 1): 866 genes were upregulated and the remaining 1,115 genes were downregulated. Tables 1,2 showed the TOP50 upregulated genes and the TOP50 downregulated genes, respectively.

\section{$G O$ analysis of the DEGs}

GO analysis contained three domains that represent gene function based on cellular component, biological process and molecular function. A total of 1,385 DEGs were associated with the cell composition domain, of which 608 were upregulated (Figure 2A) and 777 genes were downregulated (Figure 2B). The TOP5 enrichment score biological process terms were "non-membranebounded organelle", "intracellular non-membrane-bounded organelle", "cytoplasmic vesicle", "intracellular vesicle" and "cytoplasmic part". A total of 1,320 DEGs were associated with the biological process domain, of which 581 were 
Table 1 The TOP50 upregulated genes (Lnc00675 vs. si-Lnc00675)

\begin{tabular}{|c|c|c|c|c|c|}
\hline NO. & Gene symbol & Description & Probe Name & GenBank accession & Fold change \\
\hline 2 & LOC100129931 & Uncharacterized LOC100129931 & A_33_P3277883 & NR_033828 & $1,235.66$ \\
\hline 3 & CCDC64B & Coiled-coil domain containing 64B & A_33_P3335590 & NM_001103175 & 161.49 \\
\hline 4 & CEP104 & Centrosomal protein 104 kDa & A_33_P3405754 & BC050721 & 59.59 \\
\hline 6 & SFN & Stratifin & A_33_P3389286 & NM_006142 & 51.13 \\
\hline 7 & UTP18 & UTP18 small subunit processome component & A_23_P130020 & NM_016001 & 42.96 \\
\hline 8 & LINC01123 & Long intergenic non-protein coding RNA 1123 & A_33_P3228609 & NR_046110 & 42.82 \\
\hline 9 & LINC01061 & Long intergenic non-protein coding RNA 1061 & A_24_P691775 & NR_037596 & 42.68 \\
\hline 12 & EFTUD1 & $\begin{array}{l}\text { Elongation factor Tu GTP binding domain } \\
\text { containing } 1\end{array}$ & A_24_P754817 & NM_024580 & 33.30 \\
\hline 13 & MAGIX & MAGI family member, $\mathrm{X}$-linked & A_24_P66105 & NM_024859 & 31.73 \\
\hline 14 & DHRS4L1 & $\begin{array}{l}\text { Dehydrogenase/reductase SDR family member } \\
4 \text { like } 1\end{array}$ & A_33_P3359368 & NM_001277864 & 29.70 \\
\hline 15 & SHISA5 & Shisa family member 5 & A_33_P3270636 & NM_001272068 & 29.61 \\
\hline 16 & SLC51B & Solute carrier family 51 , beta subunit & A_23_P436284 & NM_178859 & 28.48 \\
\hline 17 & TBC1D31 & TBC1 domain family, member 31 & A_23_P334218 & NM_145647 & 28.18 \\
\hline 22 & SSPO & SCO-Spondin & A_33_P3277178 & AK093431 & 25.43 \\
\hline 23 & PPP1R1A & $\begin{array}{l}\text { Protein phosphatase } 1 \text {, regulatory (inhibitor) } \\
\text { subunit } 1 \mathrm{~A}\end{array}$ & A_33_P3383471 & AK123969 & 25.30 \\
\hline 24 & MAGEB6 & Melanoma antigen family $B, 6$ & A_33_P3368755 & NM_173523 & 24.11 \\
\hline 25 & BCR & Breakpoint cluster region & A_24_P127235 & NM_004327 & 24.05 \\
\hline 26 & DCLRE1B & DNA cross-link repair 1B & A_24_P54131 & NM_022836 & 23.81 \\
\hline 27 & RNF150 & Ring finger protein 150 & A_24_P350589 & NM_020724 & 23.74 \\
\hline 28 & HERC6 & $\begin{array}{l}\text { HECT and RLD domain containing E3 ubiquitin } \\
\text { protein ligase family member } 6\end{array}$ & A_33_P3315779 & NM_001165136 & 23.19 \\
\hline 29 & CUL4A & Cullin 4A & A_33_P3322909 & NM_001278513 & 23.00 \\
\hline 30 & SCOC-AS1 & SCOC antisense RNA 1 & A_24_P145019 & NR_033939 & 22.94 \\
\hline
\end{tabular}

Table 1 (continued) 
Table 1 (continued)

\begin{tabular}{|c|c|c|c|c|c|}
\hline NO. & Gene symbol & Description & Probe Name & GenBank accession & Fold change \\
\hline 32 & BTN3A1 & Butyrophilin, subfamily 3 , member A1 & A_33_P3388466 & NM_007048 & 22.47 \\
\hline 33 & AKAP12 & A kinase (PRKA) anchor protein 12 & A_23_P111311 & NM_144497 & 21.77 \\
\hline 34 & CDCA7 & Cell division cycle associated 7 & A_33_P3296169 & NM_031942 & 21.75 \\
\hline 36 & STARD13 & $\begin{array}{l}\text { StAR-related lipid transfer (START) domain } \\
\text { containing } 13\end{array}$ & A_23_P342727 & NM_178006 & 21.51 \\
\hline 37 & CTNND1 & Catenin, delta 1 & A_33_P3209716 & NM_001206885 & 21.20 \\
\hline 38 & PPAN-P2RY11 & PPAN-P2RY11 readthrough & A_33_P3239759 & NM_001198690 & 21.06 \\
\hline 42 & THOC2 & THO complex 2 & A_33_P3235690 & NM_001081550 & 20.63 \\
\hline 43 & $\mathrm{ZCCHC} 13$ & Zinc finger, $\mathrm{CCHC}$ domain containing 13 & A_32_P11096 & NM_203303 & 20.20 \\
\hline 44 & EGFR & Epidermal growth factor receptor & A_33_P3351944 & NM_201283 & 20.01 \\
\hline 45 & FBXO2 & F-box protein 2 & A_23_P45999 & NM_012168 & 19.78 \\
\hline 46 & $\mathrm{BICC} 1$ & Bicc family RNA binding protein 1 & A_33_P3293913 & NM_001080512 & 19.61 \\
\hline 47 & PCM1 & Pericentriolar material 1 & A_24_P555510 & NM_006197 & 19.56 \\
\hline
\end{tabular}

upregulated (Figure 3A) and 739 were down-regulated (Figure 3B). The TOP5 enrichment score biological process terms were "oxoacid metabolic process", "oxidationreduction process", "organic acid metabolic process", "carboxylic acid metabolic process" and "small molecule metabolic process". A total of 1,324 DEGs were associated with the molecular function domain, of which 580 were upregulated (Figure $4 A$ ) and 744 were down-regulated (Figure $4 B$ ). The five most enriched molecular function terms were "oxidoreductase activity", "protein binding", "steroid dehydrogenase activity, acting on the $\mathrm{CH}-\mathrm{OH}$ group of donors, NAD or NADP as acceptor", "protein binding" and "oxidoreductase activity, acting on paired donors, with incorporation or reduction of molecular oxygen".

\section{Pathway analysis of the DEGs}

Pathway analysis of the DEGs allows the identification of DEGs related to specific cell pathways. Pathway analysis revealed that DEGs were significantly enriched in 73 pathways (Figure $5 A, B$ ). The upregulated genes were involved in 23 pathways and the downregulated DEGs were involved in 50 pathways. The upregulated DEGs were mainly involved in "JAK-STAT signaling pathway", "Cell cycle", "Amoebiasis", "Necroptosis", "Nucleotide excision repair", "Inflammatory bowel disease (IBD)", "Adrenergic signaling in cardiomyocytes", "ErbB signaling pathway", "PI3K-Akt signaling pathway" and "Renal cell carcinoma". The downregulated DEGs were mainly involved in "Steroid biosynthesis", "Glycosaminoglycan degradation", "Adherens junction", "Lysosome, Ferroptosis", "HIF-1 signaling 
Table 2 The TOP50 downregulated genes (Lnc00675 vs. si-Lnc00675)

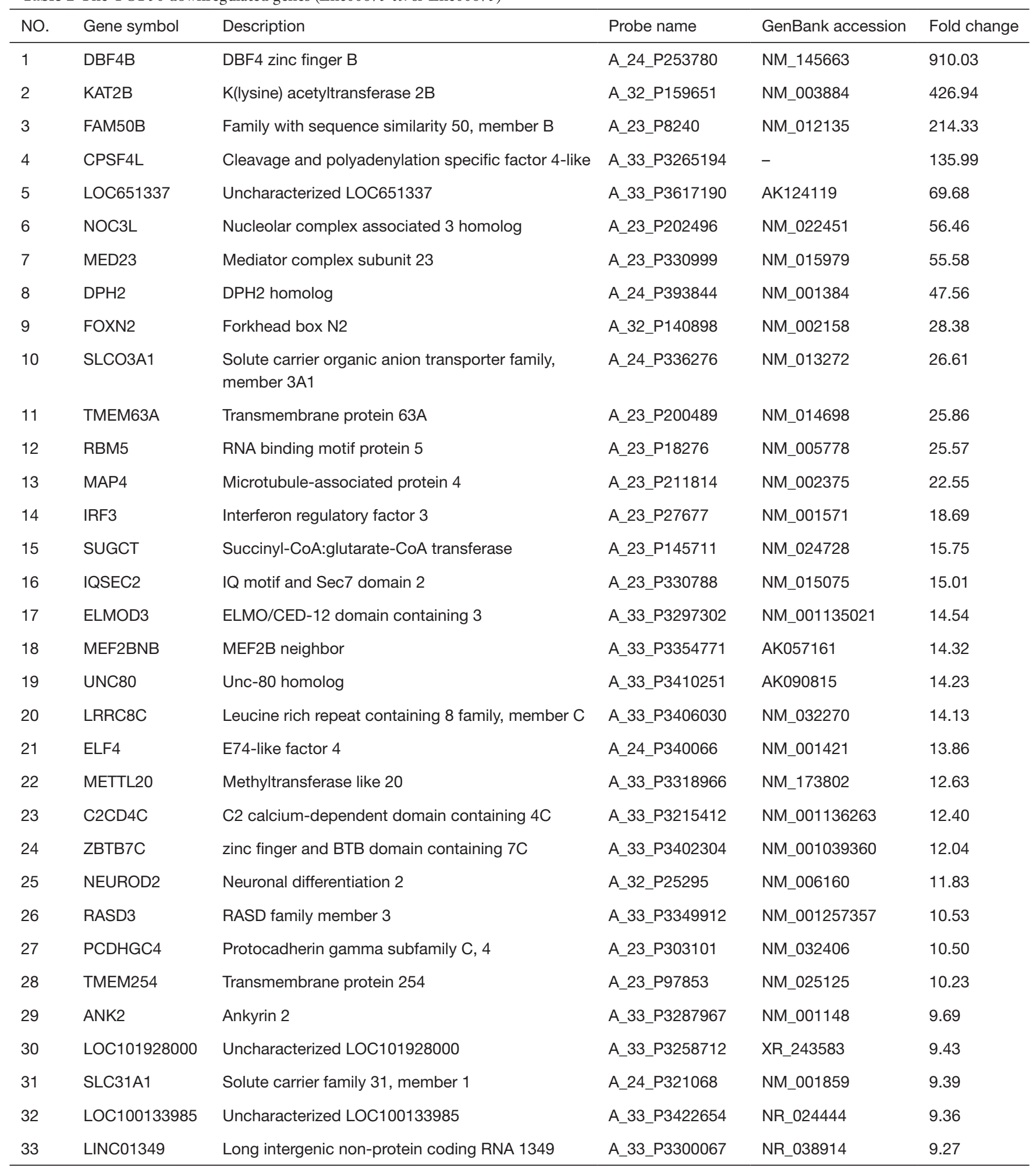

Table 2 (continued) 
Table 2 (continued)

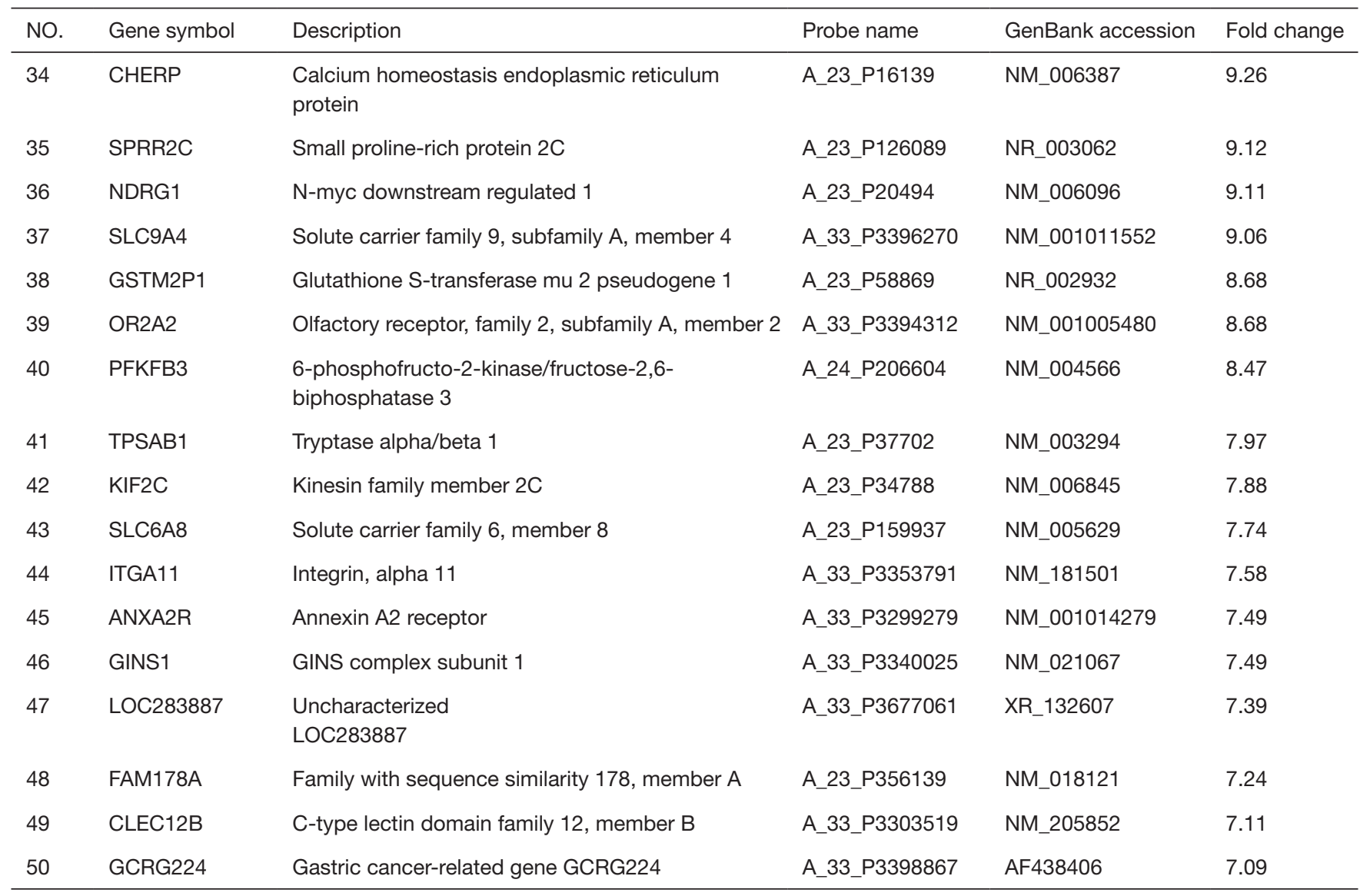

pathway", "Central carbon metabolism in cancer", "Carbon metabolism", "Glycolysis/Gluconeogenesis", "Amino sugar and nucleotide sugar metabolism" and "Fatty acid metabolism".

\section{Effects of Lnc00675 on proliferation and apoptosis in U937 cells}

The proliferation rate of si-Lnc00675 group was significantly lower than those of si-NC group and Lnc00675 group at all three time points $(\mathrm{P}<0.05)$. There was no significant difference in proliferation rate between si-NC group and Lnc00675 group $(\mathrm{P}>0.05)$ (Figure 6A). Flow cytometric analysis indicated that the downregulation of Lnc00675 significantly promoted cell apoptosis. The cell apoptosis rate of si-Lnc00675 group $(22.93 \pm 2.24)$ was significantly higher than those of si-NC group $(0.37 \pm 0.88)$ and Lnc00675 group $(0.73 \pm 0.35)(\mathrm{P}>0.01)$ (Figure $6 B)$.

\section{Discussion}

With the increasing understanding of the lncRNA, the association between tumorigenesis and lncRNA has attracted more and more attention. Notably, Multiple AML researches had shown that the high expression of lncRNA could lead to promote cell proliferation, repress apoptosis, worse prognosis and poor treatment outcomes, such as ZEB2-AS1 (14), lnc-SOX6-1 (15), lnc-CRNDE (16), lnc-HOTAIR (17). With regard to glioma, the high expression of Lnc00675 was dramatically associated with large tumor and advanced World Health Organization grade size (12). The high expression of Lnc00675 positively correlated with poor survival, perineural invasion and lymph node metastasis in patients with pancreatic ductal adenocarcinoma (11). But there is currently no research results available for correlation between Lnc00675 and AML. In the present study, we first reported that the downregulation of Lnc00675 expression 
A

GO cellular component classification

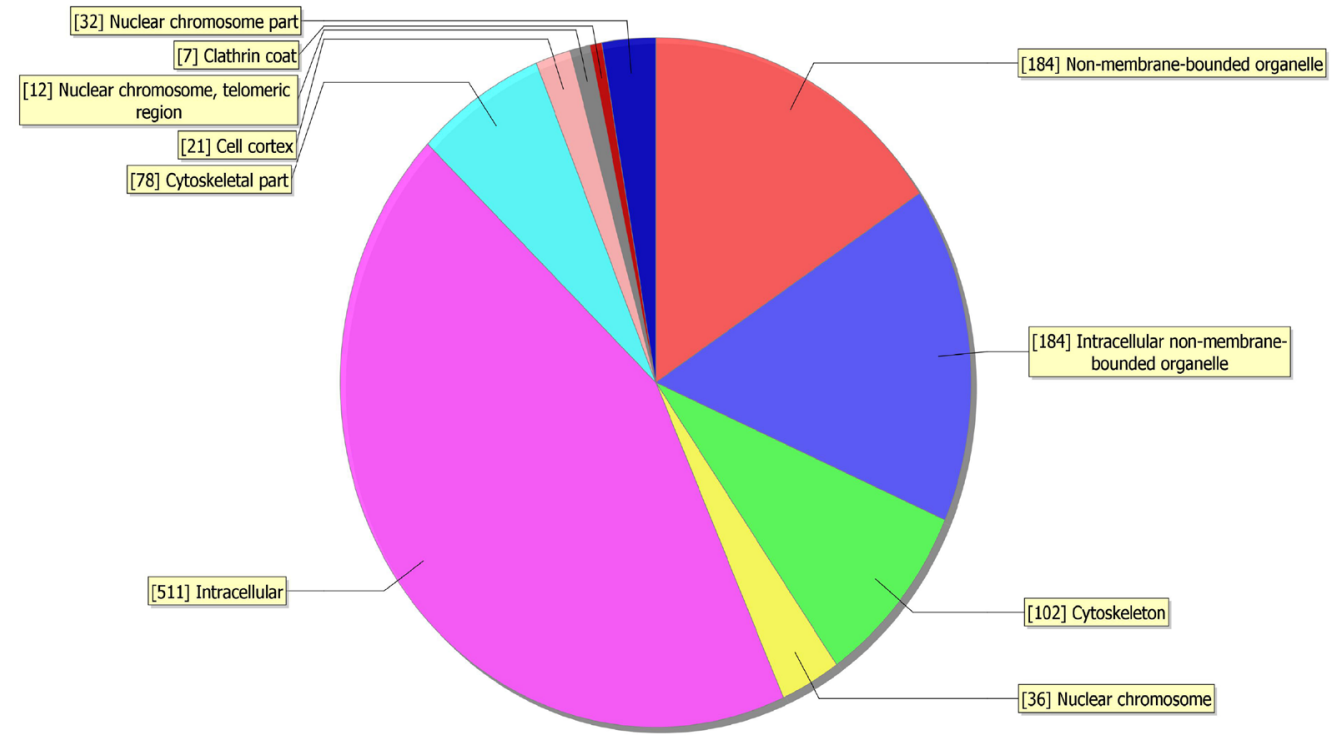

B GO cellular component classification

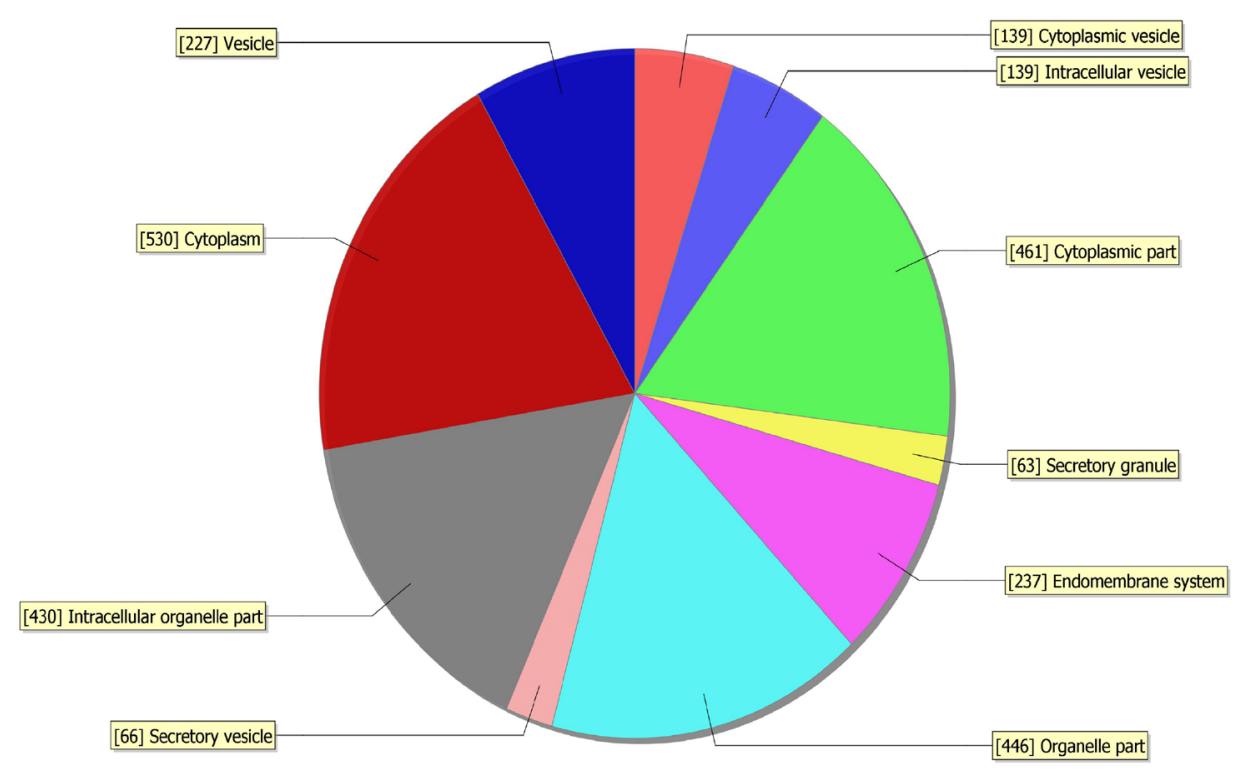

Figure 2 Gene ontology cellular component classification. (A) Cellular component classification of upregulated differentially expressed genes; (B) cellular component classification of downregulated differentially expressed genes. 
A

GO biological process classification

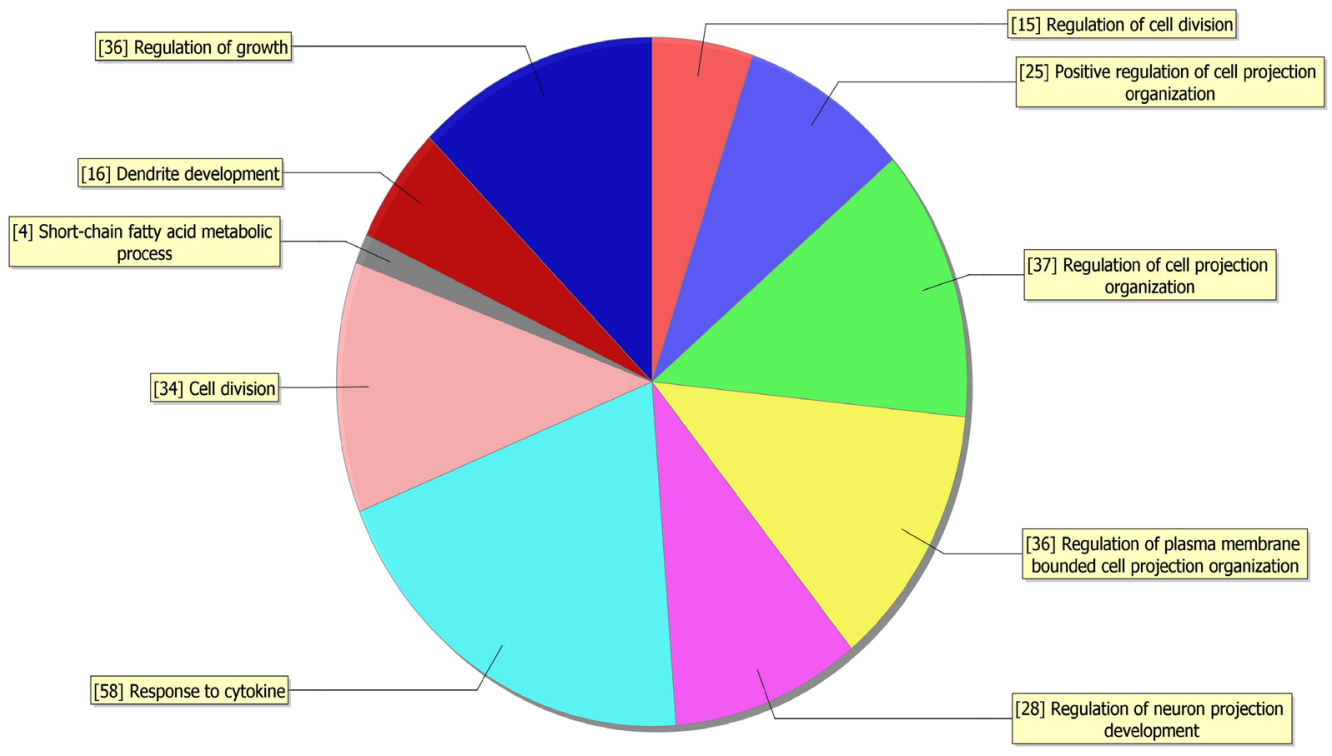

B GO biological process classification

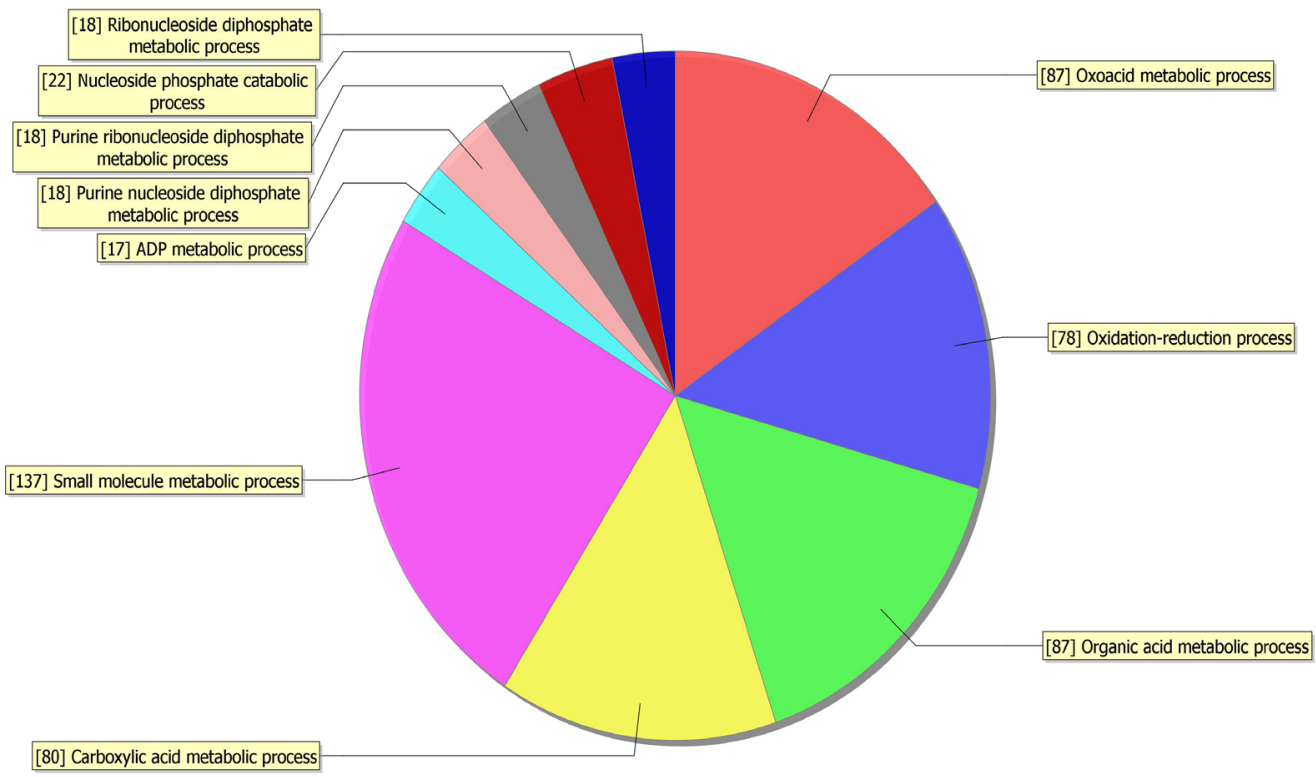

Figure 3 Gene ontology biological process classification. (A) Biological process classification of upregulated differentially expressed genes; (B) biological process classification of downregulated differentially expressed genes. 
A

GO molecular function classification

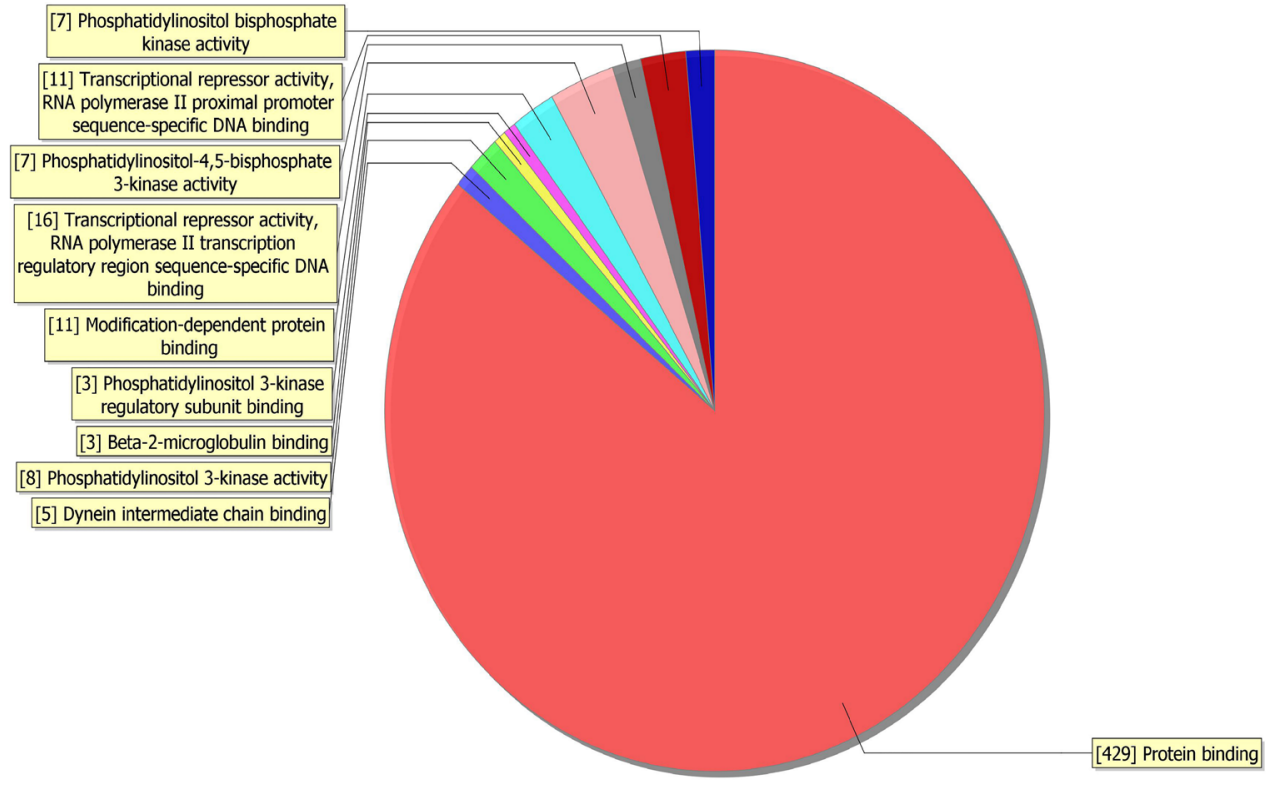

B GO molecular function classification

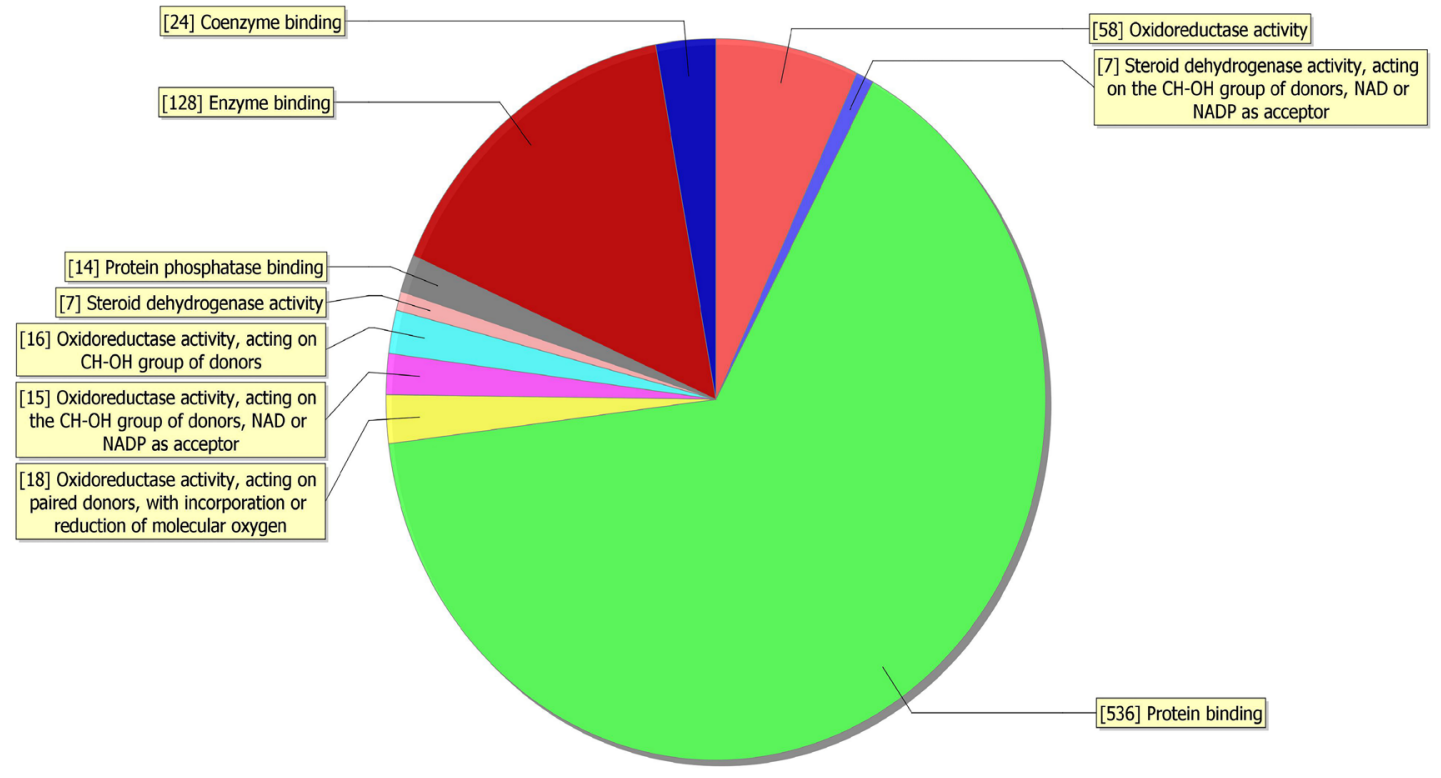

Figure 4 Gene ontology molecular function classification. (A) Molecular function classification of upregulated differentially expressed genes; (B) molecular function classification of downregulated differentially expressed genes. 
A

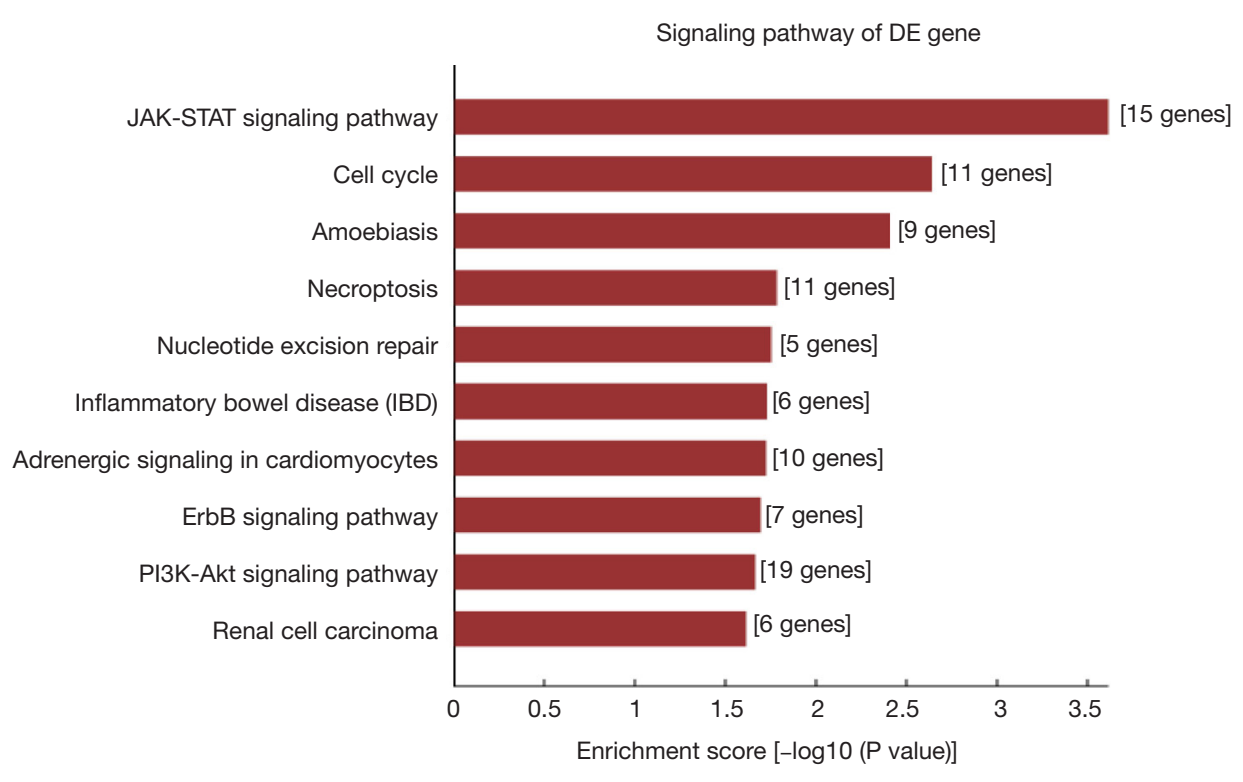

B

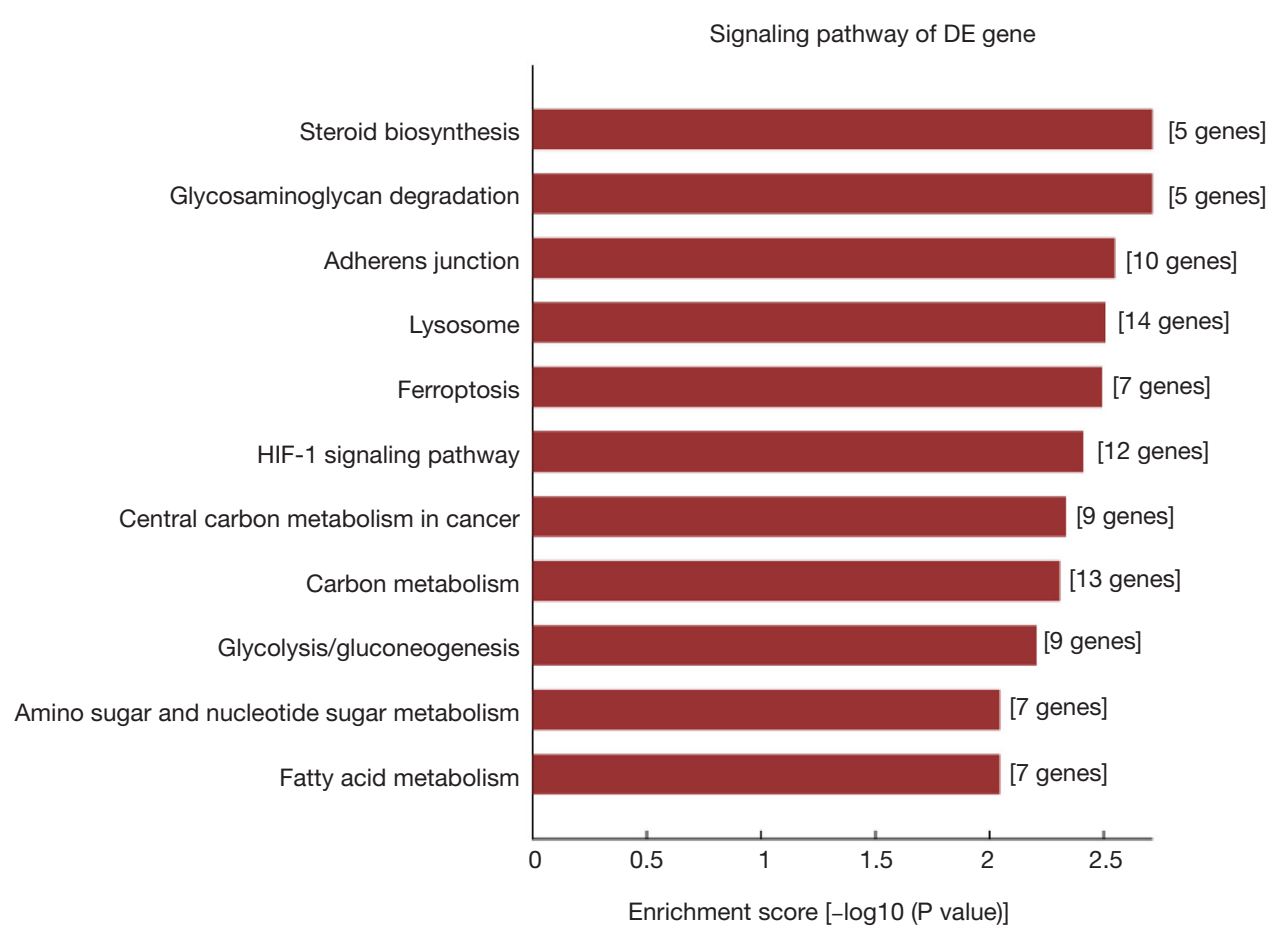

Figure 5 Significantly enrichment pathway analysis of differentially expressed (DE) genes. (A) Upregulated DE genes involved in the Top10 pathways; (B) downregulated DE genes involved in the Top10 pathways. 
A
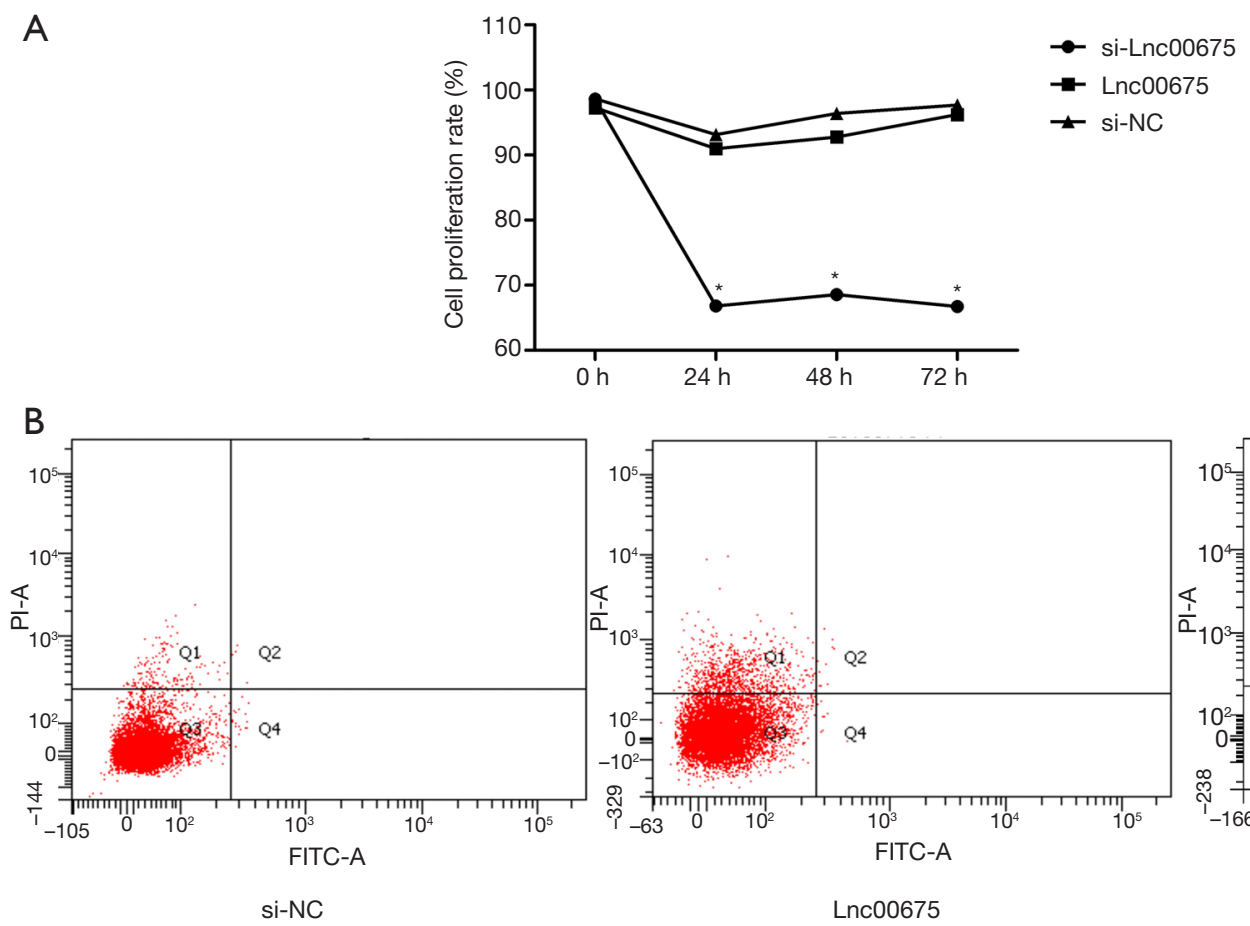

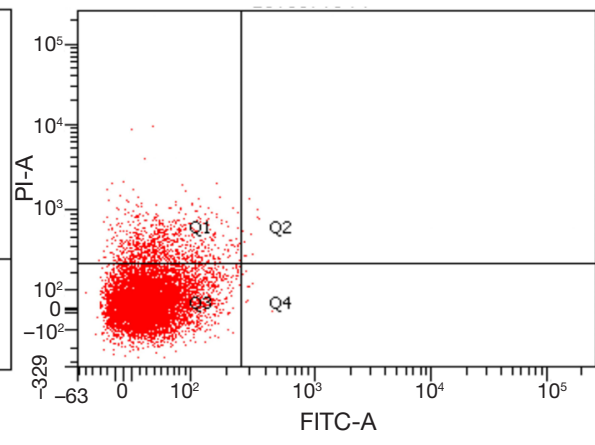

Lnc00675

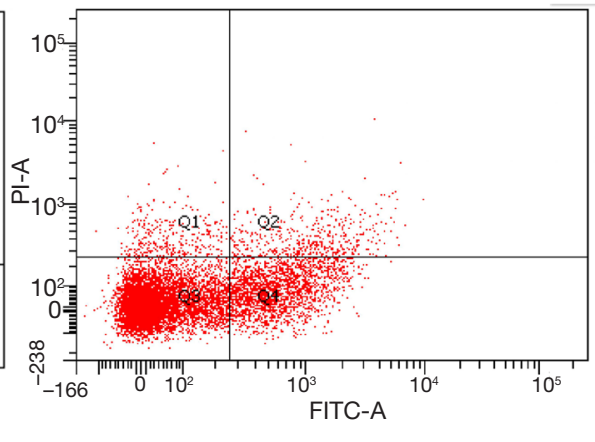

si-Lnc00675

Figure 6 Downregulation of Lnc00675 expression inhibited proliferation and induced cell apoptosis in U937 cells. (A) Cell proliferation was measured by CCK8 assay in U937 cells of Lnc00675 group, si-Lnc00675 group and si-NC group; (B) flow cytometry assay was performed to examine cell apoptosis in U937 of Lnc00675 group, si-Lnc00675 group and si-NC group. *, $\mathrm{P}<0.05$ between si-Lnc00675 group and siNC group or Lnc00675 group.

resulted in inhibiting cell proliferation and inducing cell apoptosis in U937 cells, but overexpression of Lnc00675 had no effect on the proliferation and apoptosis in U937 cells.

The Wingless $(\mathrm{Wnt}) / \beta$-catenin signaling pathway has been associated with metabolic reprogramming of cancer cells, cancer stem cells, tumorigenesis and tumor plasticity (18). Ma et al. reported that Lnc00675 inhibited apoptosis and promoted proliferation, migration and invasion though the $\mathrm{Wnt} / \beta$-catenin pathway in cervical cancer cells, and lithium chloride could attenuate the effects of Lnc00675 knockdown (13). Shan et al. revealed that Lnc00675 downregulated miR-942 expression in colorectal cancer cells, and miR-942 bound to 3'UTR of glycogen synthase kinase-3 $\beta$ (GSK-3 $\beta$, a kinase mediating $\beta$-catenin phosphorylation in $\mathrm{Wnt} / \beta$-catenin pathway) by dual-luciferase reporter assay (19). At present, there are no studies investigating the molecular mechanism of Lnc00675 in AML cells. In this regard, KEGG pathway analysis were performed using standard enrichment calculation methods to reveal the molecular mechanism. The result of pathway analysis indicated that Lnc00675 involved in JAK-STAT signaling pathway and PI3K-Akt signaling pathway. The activation of JAK-STAT signaling pathway was implicated in the pathogenesis of AML $(20,21)$, and targeting of this pathway was an effective therapeutic strategy for AML $(22,23)$. Dos Santos et al. demonstrated that the PI3KAkt signaling pathway was constitutively activated in approximately $60 \%$ of AML patients cells (24). PI3K-Akt signaling pathway inhibitors, which used alone or with other drugs, have been proven effective for suppressing cell proliferation and promoting apoptosis in AML patients, cell lines or animal models (25).

Epidermal growth factor receptor (EGFR) and interleukin 2 receptor subunit alpha (IL2RA) are involved in both JAK-STAT signaling pathway and PI3K-Akt signaling pathway. Comparing upregulation of Lnc00675 with downregulation of Lnc00675, we found that the expressions of EGFR ( $\mathrm{FC}=20.01)$ and IL2RA $(\mathrm{FC}=10.56)$ were drastically upregulated. EGFR is a cell membrane receptor tyrosine kinase, and mutant EGFR are meaningful serological markers for diagnosis of AML (26). EGFR small 
molecule inhibitors have been reported to induce complete and durable remission in AML patients (27). Researches indicated a strong association of IL2RA expression with tyrosine kinases pathways. Upregulation of IL2RA expression was correlated with upregulation expressions of fms related receptor tyrosine kinase 3 (FLT3) (28) and inhibitor of DNA binding 1 (ID1) (29), a key target of tyrosine kinases contributing to leukemia transformation. High expression of IL2RA mRNA was an independent and adverse prognostic factor in AML (30).

The present study, to best of our knowledge, was the first to reveal that downregulation of Lnc00675 expression inhibited proliferation and promoted apoptosis in human leukemia U937 cells. By comparing upregulation of Lnc00675 and downregulation of Lnc00675. We identified 866 upregulated DEGs and 1,115 downregulated DEGs, and indicated that Lnc00675 probably affected U937 cells proliferation and apoptosis through JAK-STAT signaling pathway and PI3K-Akt signaling pathway. We will elucidate molecular mechanism of Lnc00675 in AML and further validate the Lnc00675-mediated signaling pathways in our following researches. The results obtained in the current study may aid in the elucidation of molecular mechanisms of Lnc00675 in AML and contribute to the development of target therapies to treat AML.

\section{Acknowledgments}

Funding: The present study was supported by grants from the Scientific Research Project of Education Department of Liaoning Province (grant no. JC2019011).

\section{Footnote}

Data Sharing Statement: Available at http://dx.doi. org/10.21037/tcr-20-1714

Conflicts of Interest: All authors have completed the ICMJE uniform disclosure form (available at http://dx.doi. org/10.21037/tcr-20-1714). The authors have no conflicts of interest to declare.

Ethical Statement: The authors are accountable for all aspects of the work in ensuring that questions related to the accuracy or integrity of any part of the work are appropriately investigated and resolved.

Open Access Statement: This is an Open Access article distributed in accordance with the Creative Commons Attribution-NonCommercial-NoDerivs 4.0 International License (CC BY-NC-ND 4.0), which permits the noncommercial replication and distribution of the article with the strict proviso that no changes or edits are made and the original work is properly cited (including links to both the formal publication through the relevant DOI and the license). See: https://creativecommons.org/licenses/by-nc$\mathrm{nd} / 4.0 \%$.

\section{References}

1. Döhner H, Estey E, Grimwade D, et al. Diagnosis and management of AML in adults: 2017 ELN recommendations from an international expert panel. Blood 2017;129:424-47.

2. Kadono M, Kanai A, Nagamachi A, et al. Biological implications of somatic DDX41 p.R525H mutation in acute myeloid leukemia. Exp Hematol 2016;44:745-754.e4.

3. Mer AS, Lindberg J, Nilsson C, et al. Expression levels of long non-coding RNAs are prognostic for AML outcome. J Hematol Oncol 2018;11:52.

4. Morlando M, Fatica A. Alteration of Epigenetic Regulation by Long Noncoding RNAs in Cancer. Int J Mol Sci 2018;19:570.

5. Tong YS, Zhou XL, Wang XW, et al. Association of decreased expression of long non-coding RNA LOC285194 with chemoradiotherapy resistance and poor prognosis in esophageal squamous cell carcinoma. J Transl Med 2014;12:233.

6. Dykes IM, Emanueli C. Transcriptional and Posttranscriptional Gene Regulation by Long Noncoding RNA. Genomics Proteomics Bioinformatics 2017;15:177-86.

7. Zhang X, Hamblin MH, Yin KJ. The long noncoding RNA Malat1: Its physiological and pathophysiological functions. RNA Biol 2017;14:1705-14.

8. Li J, Tian H, Yang J, et al. Long Noncoding RNAs Regulate Cell Growth, Proliferation, and Apoptosis. DNA Cell Biol 2016;35:459-70.

9. Bhan A, Soleimani M, Mandal SS. Long Noncoding RNA and Cancer: A New Paradigm. Cancer Res 2017;77:3965-81.

10. Sanchez Calle A, Kawamura Y, Yamamoto Y, et al. Emerging roles of long non-coding RNA in cancer. Cancer Sci 2018;109:2093-100.

11. Li DD, Fu ZQ, Lin Q, et al. Linc00675 is a novel marker of short survival and recurrence in patients with 
pancreatic ductal adenocarcinoma. World J Gastroenterol 2015;21:9348-57.

12. Li Z, Li Y, Wang Q. LINC00675 is a prognostic factor and regulates cell proliferation, migration and invasion in glioma. Biosci Rep 2018;38:BSR20181039.

13. Ma S, Deng X, Yang Y, et al. The lncRNA LINC00675 regulates cell proliferation, migration, and invasion by affecting $W n t / \beta$-catenin signaling in cervical cancer. Biomed Pharmacother 2018;108:1686-93.

14. Shi X, Li J, Ma L, et al. Overexpression of ZEB2-AS1 lncRNA is associated with poor clinical outcomes in acute myeloid leukemia. Oncol Lett 2019;17:4935-47.

15. Guan X, Wen X, Xiao J, et al. Lnc-SOX6-1 upregulation correlates with poor risk stratification and worse treatment outcomes, and promotes cell proliferation while inhibits apoptosis in pediatric acute myeloid leukemia. Int J Lab Hematol 2019;41:234-41.

16. Wang Y, Zhou Q, Ma JJ. High expression of lnc-CRNDE presents as a biomarker for acute myeloid leukemia and promotes the malignant progression in acute myeloid leukemia cell line U937. Eur Rev Med Pharmacol Sci 2018;22:763-70.

17. El-Khazragy N, Ghozy S, Matbouly S, et al. Interaction between 12 p chromosomal abnormalities and LncHOTAIR mediated pathway in acute myeloid leukemia. J Cell Biochem 2019;120:15288-96.

18. El-Sahli S, Xie Y, Wang L, et al. Wnt Signaling in Cancer Metabolism and Immunity. Cancers (Basel) 2019;11:904.

19. Shan Z, An N, Qin J, et al. Long non-coding RNA Linc00675 suppresses cell proliferation and metastasis in colorectal cancer via acting on miR-942 and Wnt/ $\beta$-catenin signaling. Biomed Pharmacother 2018;101:769-76.

20. Cook AM, Li L, Ho Y, et al. Role of altered growth factor receptor-mediated JAK2 signaling in growth and maintenance of human acute myeloid leukemia stem cells.
Blood 2014;123:2826-37.

21. Gouilleux-Gruart V, Gouilleux F, Desaint C, et al. STATrelated transcription factors are constitutively activated in peripheral blood cells from acute leukemia patients. Blood 1996;87:1692-7.

22. Faderl S, Ferrajoli A, Harris D, et al. Atiprimod blocks phosphorylation of JAK-STAT and inhibits proliferation of acute myeloid leukemia (AML) cells. Leuk Res 2007;31:91-5.

23. Venugopal S, Bar-Natan M, Mascarenhas JO. JAKs to STATs: A tantalizing therapeutic target in acute myeloid leukemia. Blood Rev 2020;40:100634.

24. Dos Santos C, Récher C, Demur C, et al. The PI3K/Akt/ mTOR pathway: a new therapeutic target in the treatment of acute myeloid leukemia. Bull Cancer 2006;93:445-7.

25. Martelli AM, Evangelisti C, Chiarini F, et al. The phosphatidylinositol 3-kinase/Akt/mTOR signaling network as a therapeutic target in acute myelogenous leukemia patients. Oncotarget 2010;1:89-103.

26. Abdel-Aziz MM. Clinical significance of serum p53 and epidermal growth factor receptor in patients with acute leukemia. Asian Pac J Cancer Prev 2013;14:4295-9.

27. Lainey E, Sébert M, Thépot $S$, et al. Erlotinib antagonizes $\mathrm{ABC}$ transporters in acute myeloid leukemia. Cell Cycle 2012;11:4079-92.

28. Ozeki K, Kiyoi H, Hirose Y, et al. Biologic and clinical significance of the FLT3 transcript level in acute myeloid leukemia. Blood 2004;103:1901-8.

29. Tam WF, Gu TL, Chen J, et al. Id1 is a common downstream target of oncogenic tyrosine kinases in leukemic cells. Blood 2008;112:1981-92.

30. Du W, He J, Zhou W, et al. High IL2RA mRNA expression is an independent adverse prognostic biomarker in core binding factor and intermediate-risk acute myeloid leukemia. J Transl Med 2019;17:191.
Cite this article as: Miao M, Li M, Liu Z, Yang W, Wang C, Hu R. Expression profiling analysis reveals molecular mechanism of Lnc00675 downregulation promoting cell apoptosis in acute myeloid leukemia U937 cells. Transl Cancer Res 2020;9(11):6867-6880. doi: 10.21037/tcr-20-1714 